\title{
Fibrinogen Is A Coagulation Marker Associated With The Prognosis Of Endometrial Cancer
}

This article was published in the following Dove Press journal: OncoTargets and Therapy

\author{
Qing $\mathrm{Li}^{\prime}$ \\ Rong Cong' \\ Fanfei Kong' \\ Jian $\mathrm{Ma}{ }^{\prime}$ \\ Qijun $W u \mathbb{D}^{2}$ \\ Xiaoxin $\mathrm{Ma}^{\mathrm{I}}$ \\ 'Department of Obstetrics and \\ Gynecology, Shengjing Hospital of China \\ Medical University, Shenyang, Liaoning, \\ People's Republic of China; ${ }^{2}$ Department \\ of Clinical Epidemiology, Shengjing \\ Hospital of China Medical University, \\ Shenyang, Liaoning, People's Republic of \\ China
}

Correspondence: Xiaoxin $\mathrm{Ma}$ Department of Obstetrics and Gynecology, Shengjing Hospital of China Medical University, No.36, SanHao Street, Shenyang, Liaoning I I0004, People's

Republic of China

$\mathrm{Tel}+8618904001666$

Email maxiaoxin666@aliyun.com
Purpose: The present study aimed to identify coagulation markers with prognostic value in the setting of surgically treated endometrial cancer.

Patients and methods: A total of 942 patients with endometrial cancer who underwent surgery were included in the study. The preoperative prothrombin time (PT), activated partial thromboplastin time (aPTT), thrombin time (TT), prothrombin time activity (PTA), fibrinogen and D-dimer values were analyzed to determine their potential associations with clinicopathological characteristics. Survival analysis was performed using the Kaplan-Meier method, p-values were calculated using the log-rank text, and the prognostic factors were evaluated using Cox's proportional hazards regression model.

Results: The preoperative plasma fibrinogen and D-dimer concentrations were significantly different among patients with different ages, pre/post-menopausal status, International Federation of Obstetrics and Gynecology Association (FIGO) stage, tumor grade, depth of myometrial invasion, histological type and lymphatic vessel space invasion. Fibrinogen level was also asscoiated with body mass index (BMI) and comorbidities, and D-dimer level was asscoiated with preoperative radiotherapy and chemotherapy. APTT was different in patients in pre/post-menopausal status and with or without comorbidities. PTA was asscoiated with BMI and lymphovascular invasion. TT was different between different age groups, different menopause status groups, as well as different FIGO stage groups. A multivariate analysis identified high fibrinogen levels $(>3.25 \mathrm{~g} / \mathrm{L})$ as an independent prognostic factor for overall survival (HR=1.807; 95\% CI=1.003-3.253; $\mathrm{p}=0.049$ ).

Conclusion: High pretreatment fibrinogen levels are associated with poor overall survival and represent a valuable independent prognostic factor in patients with endometrial cancer. PT, aPTT, TT, PTA and D-dimer levels cannot be used as independent prognostic factors for endometrial cancer.

Keywords: endometrial carcinoma, hemostatic markers, clinicopathological characteristics, survival

\section{Introduction}

Endometrial cancer (EC) is one of the most common malignant tumors in the female reproductive system and accounts for $20 \%$ to $30 \%$ of female reproductive tract tumors; moreover, the global incidence of EC is increasing year to year. EC is the most common gynecologic malignancy in Europe and the United States and is the second most common gynecological malignancy in China. ${ }^{1,2}$ Assessing the progression and prognosis of EC primarily relies on invasive surgery and pathology, ${ }^{3}$ and effective biomarkers that can assess disease status are still needed.

Some studies have found that patients with malignant tumors may have abnormal hemostasis. One study has shown that patients with glioblastoma had plasma 
hypercoagulability. ${ }^{4}$ Zhang et $\mathrm{al}^{5}$ believed that hemostasis parameters can be used to predict the prognosis of patients with advanced pancreatic cancer. The coagulation cascade activation mechanism often occurs in patients with malignant tumors. Vascular endothelial growth factor (VEGF-A), which is secreted by tumor cells, indirectly promotes coagulation and changes the coagulation properties of endothelial cells. ${ }^{6,7}$ The leakage of plasma proteins from the tumor vascular endothelium induces inflammatory responses and exogenous coagulation pathways, ${ }^{8,9}$ and tumor cells release procoagulant tissue factor and tumor necrosis factor. ${ }^{10}$

Although many studies have found a link between hemostasis markers and malignant tumors, the relationship between hemostatic markers and EC survival outcomes is unclear. To assess the relationship between preoperative hemostatic markers and clinicopathological parameters in patients with EC and identify hemostatic markers that affect the survival of patients with postoperative EC, we performed a retrospective study at Shengjing Hospital of China Medical University.

\section{Materials And Methods}

\section{Ethical Statement}

The retrospective study was approved by the Institutional Review Board of the Ethics Committee of Shengjing Hospital of China Medical University (No. 2017PS292K) and was carried on in accordance with the principles of the Declaration of Helsinki. All patients have provided written informed consents in the study.

\section{Patients}

A total of 1165 patients were diagnosed with EC at Shengjing Hospital of China Medical University between January 2012 and March 2017. All patients included in the study met the following criteria: age $>18$ years old; underwent hysterectomy at Shengjing Hospital; postoperative pathological diagnosis of EC; no history of other malignant tumors; no active infection; no blood disease or other serious comorbidities; no thromboembolic events; and all needed variables in this study are complete. A total of 223 patients were excluded due to not meeting the inclusion criteria, and 942 patients were included in the present study.

\section{Methods}

We collected the following information from electronic medical records from the hospital information system of Shengjing Hospital: patient's age at diagnosis, preoperative body mass index (BMI), pre/post-menopausal status, International Federation of Obstetrics and Gynecology Association (FIGO) stage, tumor grade, histology type, tumor myometrial invasion depth, lymphatic vascular invasion, whether radiotherapy and chemotherapy occurred before surgery and whether comorbidities occurred, such as diabetes, hypertension and hyperlipidemia.

The above data were collected by experienced gynecologists and pathologists. The WHO classification was used to classify the tumors, and all tumors were clinically staged according to FIGO guidelines (2009). Pathologists divide tumors into well (G1), moderately (G2) or poorly (G3) differentiated, and the histology was distinguished as type I (mainly endometrioid) or type II (non-endometrioid).

Peripheral blood was drawn from the patient within 15 days before surgery and examined for the following coagulation markers: prothrombin time (PT), activated partial thromboplastin time (aPTT), thrombin time (TT), prothrombin time activity (PTA), fibrinogen and D-dimer. Blood samples were collected in a vacuum blood collection tube and mixed with a $0.109 \mathrm{mmol} / \mathrm{L}$ sodium citrate anticoagulant (in a 9:1 ratio). The specimen was centrifuged with automatic balance centrifuge (Type DT5-3, low-speed automatic balance centrifuge, Beijing Times Beili Centrifuge Co., Ltd.) at $1500 \mathrm{~g}$ for $15 \mathrm{~min}$ at $20-25^{\circ} \mathrm{C}$ to obtain platelet-poor plasma, which was immediately evaluated by a CA-1500 automatic coagulometer (Sysmex America, Inc). PT, aPTT and TT were evaluated using the Thrombor $\mathrm{S}^{\circledR}$ (Dade Behring $\mathrm{GmbH}$, Germany), Dade Actin FS ${ }^{\circledR}$ (Dade Behring GmbH, Germany) and TestThrombin Reagent ${ }^{\circledR}$ (Dade Behring GmbH, Germany) commercial kits, respectively. Fibrinogen and D-dimer were detected using a commercial Iatron kit (Mitsubishi Kagaku Iatron, Inc., Tokyo, Japan).

The patients were followed up after surgery by telephone. The last follow-up date was June 30, 2018. The primary point of the analysis was overall survival (OS), which was defined as the time between surgery and death from any cause or the date of the last follow-up for patients who were still alive. The date of death was acquired by follow-up or from the death certificate.

\section{Statistical Analysis}

All statistical calculations were performed using SPSS software (version 19.0; IBM SPSS, Inc., Chicago, IL, USA). Categorical variables were expressed as numbers and percentages, while continuous variables were represented by the median with an interquartile range (IQR). The MannWhitney $U$-test was used to assess the significance of 
between-group differences. The coagulation marker cut-off values were determined by the receiver operating characteristic (ROC) curve and were set as the values that had the highest sensitivity and specificity. The Kaplan-Meier method was applied to construct survival curves, and the log-rank text was used to compare different groups. Cox proportional hazards regression was used to perform univariate and multivariate analyses to identify prognostic factors and estimate the hazard ratios (HRs) and 95\% confidence intervals (CIs). A P value of less than 0.05 is considered to be statistically significant.

\section{Results}

\section{Patient Characteristics}

Figure 1 shows the flow chart of the patient inclusion and exclusion process in this study, a total of 942 patients were included in the final analysis. The median age of the patients was 56 years (IQR: 51 to 62), the median preoperative BMI of the patients was $25.12 \mathrm{~kg} / \mathrm{m}^{2}$ (IQR: 23.19 to 27.74 ) and the median follow-up time was 43 months (IQR: 28 to 56 months). The clinicopathological characteristics of the patients are summarized in Table 1.

\section{Relationships Between Clinical} Characteristics And Hemostatic Markers Of the examined coagulation markers, fibrinogen and D-dimer levels were significant different in groups with pre/post-menopausal status, different ages, FIGO stage, grade, depth of myometrial invasion, histological type and lymphovascular invasion, fibrinogen level was also different in patients who were and were not overweight and presented comorbidities, and the D-dimer level was different in patients received radiotherapy and chemotherapy or not before surgeries (p-values are shown in Table 2). The aPTT value was different in patients in pre/post-menopausal status and with or without comorbidities $(\mathrm{p}=0.006$ and $\mathrm{p}=0.035$, respectively). Significant differences in the PTA levels were observed between patients with higher BMI and normal

\section{Endomertial cancer diagnosed in 2012-2017 at Shengjing Hospital of China Medical University} $(\mathrm{N}=1165)$

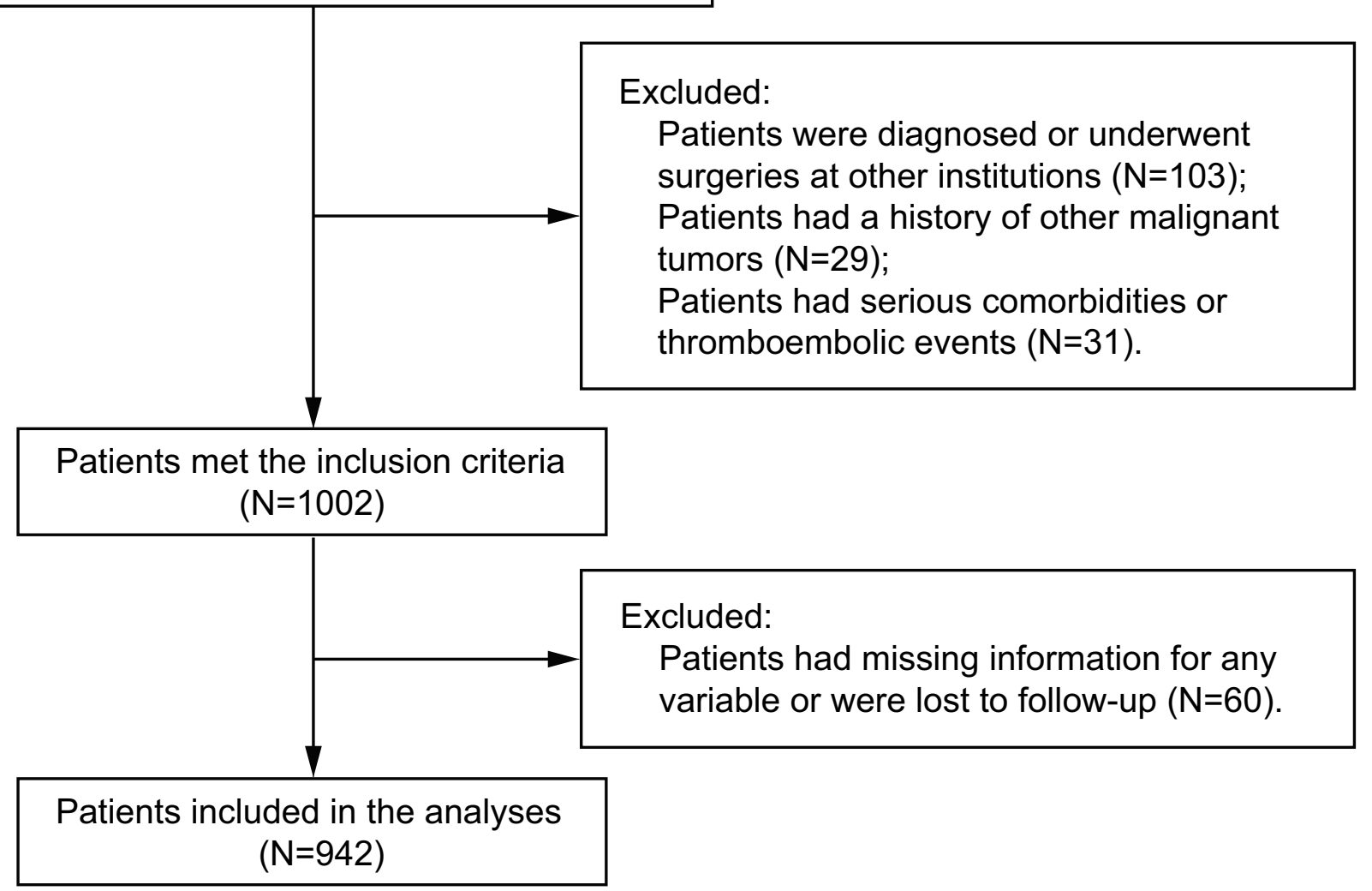

Figure I Flow chart of the patient inclusion and exclusion process. 
Table I Patient Characteristics

\begin{tabular}{|c|c|c|}
\hline Characteristics & No. Of Patients (\%) & $\begin{array}{l}\text { Survival/ } \\
\text { Death }\end{array}$ \\
\hline \multicolumn{3}{|l|}{ Age at diagnosis, $y r$} \\
\hline$<60$ & $620(65.82 \%)$ & $590 / 30$ \\
\hline$\geq 60$ & $322(34.18 \%)$ & $298 / 24$ \\
\hline \multicolumn{3}{|l|}{ BMI, $\mathrm{kg} / \mathrm{m}^{2}$} \\
\hline$<24$ & 323 (34.29\%) & $308 / 15$ \\
\hline$\geq 24$ & $619(65.71 \%)$ & $578 / 39$ \\
\hline \multicolumn{3}{|l|}{ Menopause } \\
\hline No & $27 \mid(28.77 \%)$ & $263 / 8$ \\
\hline Yes & $67 \mid(71.23 \%)$ & $625 / 46$ \\
\hline \multicolumn{3}{|l|}{ FIGO stage } \\
\hline I & 758 (80.47\%) & $729 / 29$ \\
\hline II/III/IV & $184(19.53 \%)$ & $159 / 25$ \\
\hline \multicolumn{3}{|l|}{ Grade } \\
\hline GI & 427 (45.33\%) & $415 / 12$ \\
\hline G2/G3 & $515(54.67 \%)$ & $473 / 42$ \\
\hline \multicolumn{3}{|l|}{ Myometrial invasion } \\
\hline$<I / 2$ & 724 (76.86\%) & $695 / 29$ \\
\hline$\geq 1 / 2$ & $218(23.14 \%)$ & $193 / 25$ \\
\hline \multicolumn{3}{|l|}{ Histological type } \\
\hline Type I & 907 (96.28\%) & $862 / 45$ \\
\hline Type II & 35 (3.72\%) & $26 / 9$ \\
\hline \multicolumn{3}{|l|}{ Lymphovascular invasion } \\
\hline Negative & $910(96.60 \%)$ & $860 / 50$ \\
\hline Positive & $32(3.40 \%)$ & $28 / 4$ \\
\hline \multicolumn{3}{|l|}{ Comorbidities } \\
\hline No & $5 I 5(54.67 \%)$ & $492 / 23$ \\
\hline Yes & 427 (45.33\%) & $396 / 31$ \\
\hline \multicolumn{3}{|l|}{ Radiotherapy or chemotherapy } \\
\hline No & 931 (98.83\%) & $878 / 53$ \\
\hline Yes & II (I.17\%) & $10 / 1$ \\
\hline
\end{tabular}

Abbreviation: FIGO, International Federation of Gynecology and Obstetrics.

BMI, and with lymphovascular invasion and those with no lymphovascular invasion $(\mathrm{p}=0.001, \mathrm{p}=0.042$, respectively). The TT levels were different among the different age groups, different menopause status groups, as well as different FIGO stage groups ( $<<0.001, \mathrm{p}<0.001$ and $\mathrm{p}<0.001$, respectively). However, across all clinicopathological parameters, there were no significant differences detected in PT.

\section{Univariate And Multivariate Analyses Of The OS Factors In Patients With EC}

To clarify the association of each factor with OS, a univariate analysis was performed with age, BMI, menopause, FIGO stage, grade, myometrial invasion, histology, lymphovascular invasion, comorbidities, radiotherapy or chemotherapy, fibrinogen level, D-dimer level, aPTT, PT, PTA and TT. The patients were divided into two groups by their clinicopathological characteristics, as shown in Table 1, using cut-off values for the hemostatic markers. According to the univariate analysis, patients with a high preoperative fibrinogen $(>3.25 \mathrm{~g} / \mathrm{L})$ level, high D-dimer $(>111.5 \mu \mathrm{g} / \mathrm{L})$ level, low PTA $(\leq 106.5 \%)$ and low TT $(\leq 14.85 \mathrm{~s})$ showed significantly lower survival than those with a low fibrinogen level, low D-dimer level, high PTA and high TT $(\mathrm{p}<0.001, \mathrm{p}<0.001$, $\mathrm{p}=0.017$, and $\mathrm{p}<0.001$, respectively). The patient survival curves were constructed according to the high and low levels of fibrinogen, D-dimer, PTA, TT by the Kaplan-Meier method and compared using a log rank test (Figure 2). Patients with post-menopausal status, FIGO stages II-IV, poor differentiation, deep myometrial invasion and nonendometrioid cancer had a poor prognosis $(\mathrm{p}=0.023$, $\mathrm{p}<0.001, \mathrm{p}=0.001, \mathrm{p}<0.001$, and $\mathrm{p}<0.001$, respectively). The HRs, 95\% CIs and p-values are shown in Figure 3.

Based on the univariate analysis results for OS factors, the fibrinogen level, D-dimer level, PTA, TT, menopause, FIGO stage, grade, myometrial invasion and histological type were entered into the Cox proportional hazards regression as covariates. The multivariate analysis showed that the high plasma fibrinogen level $(>3.25 \mathrm{~g} / \mathrm{L}) \quad(\mathrm{HR}=1.807$; 95\% CI $=1.003-3.253 ; \mathrm{p}=0.049)$, post-menopausal status $(\mathrm{HR}=2.197 ; 95 \% \mathrm{CI}=1.012-4.691 ; \mathrm{p}=0.047)$ and histological type (type II ) $(\mathrm{HR}=2.516 ; 95 \% \mathrm{CI}=1.175-5.387$; $\mathrm{p}=0.018$ ) were independent risk factors for OS. The p-values, HRs and 95\% CIs are shown in Figure 4.

\section{Discussion}

In previous studies, patients with malignant tumors often had coagulation dysfunction, which manifested as a hypercoagulable state in patients. ${ }^{11,12}$ Tumor cells and tumor-associated inflammatory cells may activate the coagulation cascade, ${ }^{13,14}$ and there are interactions between hemostatic factors and tumor biology. ${ }^{15}$ In this study, we first estimated the prognostic value of six coagulation markers in patients with EC and found that the preoperative plasma fibrinogen level is an independent prognostic factor for EC.

Although most patients were diagnosed early due to obvious clinical manifestations, such as abnormal vaginal bleeding or drainage in the early stage of EC, some patients still had a poor prognosis. The clinicopathological characteristics of EC, including patient age, clinical stage, 
Table 2 Relationships Between The Clinicopathological Characteristics And Hemostatic Markers

\begin{tabular}{|c|c|c|c|c|c|c|}
\hline \multirow[t]{2}{*}{ Characteristics } & \multicolumn{6}{|l|}{$P$ value } \\
\hline & Fibrinogen & $\begin{array}{l}\text { D- } \\
\text { dimer }\end{array}$ & aPTT & PT & PTA & TT \\
\hline $\begin{array}{l}\text { Age at diagnosis, } \\
\begin{array}{l}\mathrm{yr} \\
\quad<60 \\
\geq 60\end{array}\end{array}$ & $<0.001$ & $<0.001$ & 0.451 & 0.927 & 0.508 & $<0.001$ \\
\hline $\begin{array}{l}\text { BMI, } \mathrm{kg} / \mathrm{m}^{2} \\
\quad<24 \\
\geq 24\end{array}$ & 0.001 & 0.454 & 0.101 & 0.688 & 0.001 & 0.142 \\
\hline $\begin{array}{l}\text { Menopause } \\
\text { No } \\
\text { Yes }\end{array}$ & 0.002 & 0.015 & 0.006 & 0.756 & 0.239 & $<0.001$ \\
\hline $\begin{array}{l}\text { FIGO stage } \\
\text { I } \\
\text { II /III/IV }\end{array}$ & 0.009 & $<0.001$ & 0.262 & 0.37 & 0.05 & $<0.001$ \\
\hline $\begin{array}{l}\text { Grade } \\
\text { GI } \\
\text { G2/G3 }\end{array}$ & 0.003 & $<0.001$ & 0.589 & 0.633 & 0.703 & 0.061 \\
\hline $\begin{array}{l}\text { Myometrial } \\
\text { invasion } \\
<1 / 2 \\
\geq 1 / 2\end{array}$ & 0.001 & $<0.001$ & 0.292 & 0.468 & 0.184 & $0.44 I$ \\
\hline $\begin{array}{l}\text { Histological type } \\
\text { Type I } \\
\text { Type II }\end{array}$ & 0.001 & $<0.001$ & 0.051 & 0.218 & 0.225 & 0.419 \\
\hline $\begin{array}{l}\text { Lymphovascular } \\
\text { invasion } \\
\text { Negative } \\
\text { Positive }\end{array}$ & 0.003 & 0.001 & 0.617 & 0.744 & 0.042 & 0.104 \\
\hline $\begin{array}{l}\text { Comorbidities } \\
\text { No } \\
\text { Yes }\end{array}$ & 0.037 & 0.247 & 0.035 & 0.809 & 0.379 & 0.477 \\
\hline $\begin{array}{l}\text { Radiotherapy or } \\
\text { chemotherapy } \\
\text { No } \\
\text { Yes }\end{array}$ & 0.351 & 0.001 & 0.819 & 0.211 & 0.305 & 0.384 \\
\hline
\end{tabular}

Abbreviations: FIGO, International Federation of Gynecology and Obstetrics aPTT, activated partial thromboplastin time; PT, prothrombin time; PTA, prothrombin activity; TT, thrombin time.

tumor grade, histological type, lymphatic vessel space invasion, etc., have been fully studied previously, and these characteristics can be considered independent risk factors for EC. ${ }^{16}$ However, these clinicopathological characteristics are obtained by invasive methods. Research efforts are focused on predicting the prognosis of EC using blood biomarkers, and coagulation markers appear to be a series of valuable indicators.
In this study, we explored the significance of betweengroup differences in coagulation markers and found that the fibrinogen level was obviously different among groups with different ages, BMI, menopause, FIGO stage, grade, myometrial invasion depth, histological type, lymphovascular invasion and comorbidities. The patients were divided into two groups according to the values calculated by the ROC curves. The Kaplan-Meier method was used to compare the survival of the two groups. A high fibrinogen concentration $(>3.25 \mathrm{~g} / \mathrm{L})$, high D-dimer concentration $(>111.5 \mu \mathrm{g} / \mathrm{L})$, low PTA level $(\leq 106.5 \%)$ and low TT level $(\leq 14.85 \mathrm{~s})$ were found to be poor prognostic factors for $\mathrm{EC}$ in the univariate analysis. However, the multivariate analysis of all prognostic factors revealed that of the six coagulation markers, only the preoperative plasma fibrinogen concentration (PFC) ( $\mathrm{HR}=1.807 ; 95 \% \mathrm{CI}=1.003-3.253 ; \mathrm{p}=0.049)$ was an independent prognostic factor for EC. Additionally, the univariate analysis showed that the menopause, FIGO stage, tumor grade, pathological type and depth of myometrial invasion were also significant prognostic factors.

Fibrinogen, also called clotting factor I, is a glycoprotein synthesized by hepatocytes that is an important substance in the process of blood coagulation and thrombosis. Several studies have suggested that elevated fibrinogen levels are associated with tumor cell growth, proliferation, migration, and apoptosis in patients with malignant tumors. ${ }^{17,18}$ Palumbo et $\mathrm{al}^{19}$ reported that fibrinogen facilitates the formation of tumor stroma and the adhesion of circulating cancer cells to the vasculature, thereby increasing the risk of cancer metastasis. Fibrinogen binds to cell growth factors like VEGF-A, promotes angiogenesis in cancer tissues, and causes cancer growth and metastasis. ${ }^{20}$ Fibrinogen also enhances the chemotactic properties of stromal cells, including endothelial cells, and promotes tumor cell migration. ${ }^{21}$

Fibrinogen levels are associated with the prognosis of patients with a variety of cancers. A multivariate analysis found that the preoperative fibrinogen to albumin ratio (FAR) is a significant independent prognostic factor for breast cancer $(\mathrm{HR}=2.622 ; 95 \% \mathrm{CI}=1.455-4.724 ; \mathrm{p}=0.001)$. Compared with the low FAR group, the patients with high FAR had worse prognosis. The effect of FAR predicting the prognosis of patients with stage II/III subgroup and luminal A-like subtypes was more prominent. ${ }^{22} \mathrm{Zhu}$ et $\mathrm{al}^{23}$ conducted a multivariate Cox proportional hazard regression model analysis and concluded that a fibrinogen level $\geq 3.295 \mathrm{~g} / \mathrm{L}$ was an independent prognostic factor for early postoperative death after bladder cancer-assisted radical cystectomy and it may 
a

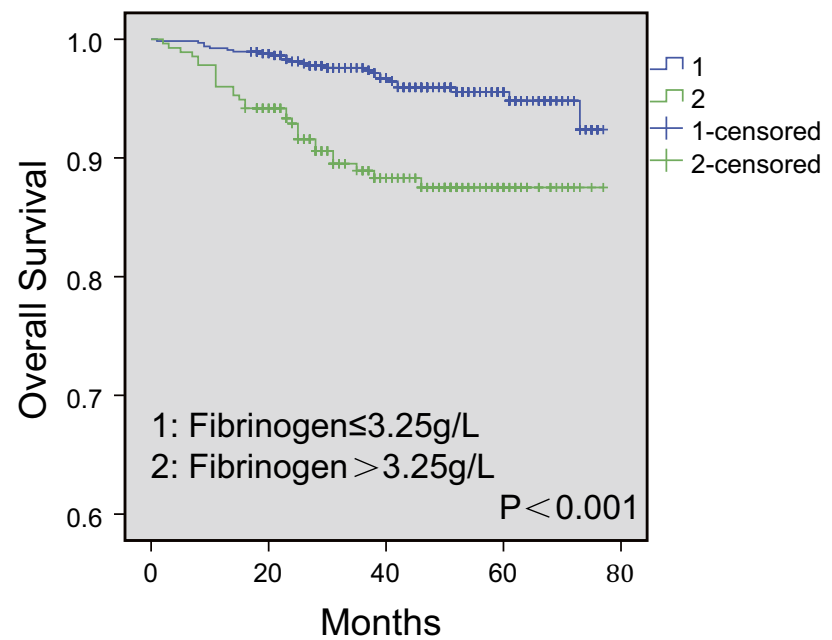

C

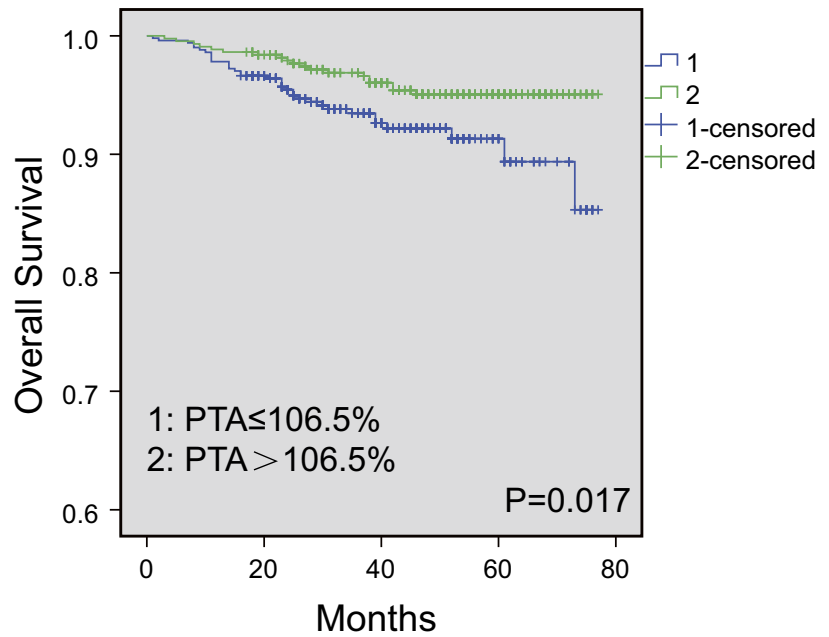

b
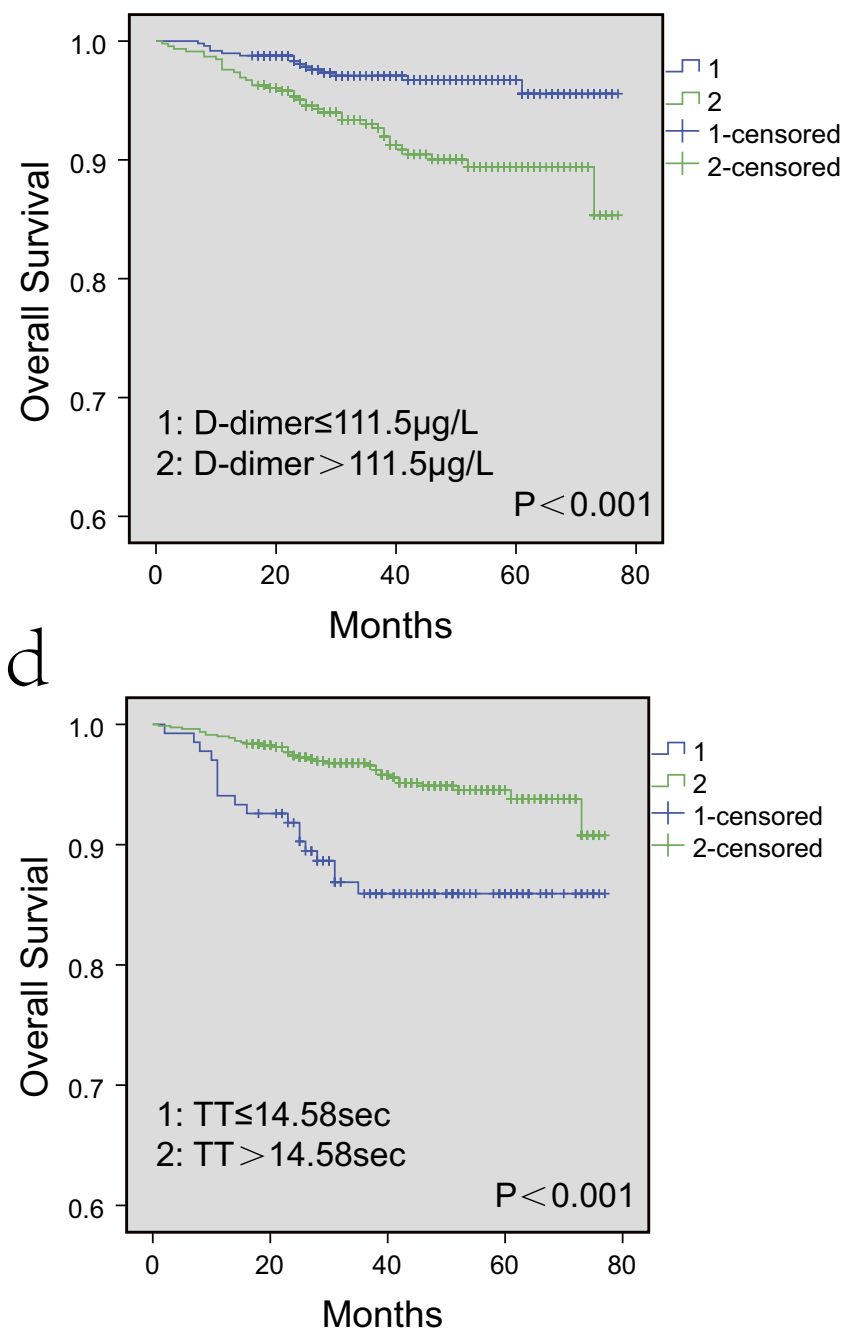

Figure 2 Kaplan-Meier survival estimates for patients with EC.

Notes: (A): Fibrinogen levels and overall survival; (B): D-dimer levels and overall survival; (C): PTA levels and overall survival; (D): TT levels and overall survival.

be considered that plasma fibrinogen is a biomarker to help guide the treatment of patients with bladder cancer. In a study of gastric cancer, patients with tumor-node-metastasis (TNM) stages I-II had lower OS rates and cancer-specific survival rates, and the plasma fibrinogen level was suggested to be an independent prognostic parameter for survival for stage I and T1-4aN0 gastric cancer. The combination of the preoperative plasma fibrinogen level and American Joint Committee on Cancer (AJCC) stage can more accurately predict the survival of patients with stages I-II gastric cancer after therapeutic surgery than either factor alone. ${ }^{24}$ Using immunohistochemistry and RT-PCR, Uccella et $\mathrm{al}^{25}$ found that EC cells can express fibrinogen. Zhou et $\mathrm{al}^{26}$ considered that a Plasma fibrinogen concentration $\geq 2.58 \mathrm{mg} / \mathrm{dL}$ can predict positive lymphovascular space invasion (LVSI) in EC; Seebacher et $\mathrm{al}^{27}$ reported that $\mathrm{PFC} \geq 388.9 \mathrm{mg} / \mathrm{dL}$ retained significance as a poor prognosticator of disease-free survival (DFS) and OS. They also found that a higher plasma fibrinogen level was associated with an advanced FIGO stage, non-endometrioid histology and higher patients' age. In another multicenter study of EC, plasma fibrinogen level was associated with higher patient age, higher BMI, obesity, deep myometrial invasion and upstaging, and PFC could be considered an independent determinant of extrauterine disease $(\mathrm{OR}=2.7$, 95\% $\mathrm{CI}=1.3-5.6)$ and an independent prognostic factor for both DFS (HR $=2.0,95 \% \mathrm{CI}=1.1-3.6)$ and $\mathrm{OS}(\mathrm{HR}=2.7,95 \%$ $\mathrm{CI}=1.3-5.5)$ in patients with $\mathrm{EC}^{28}$

In our study, the preoperative plasma fibrinogen level was associated with age, BMI, pre/post-menopausal status, FIGO stage, myometrial invasion, histological type, lymphovascular 


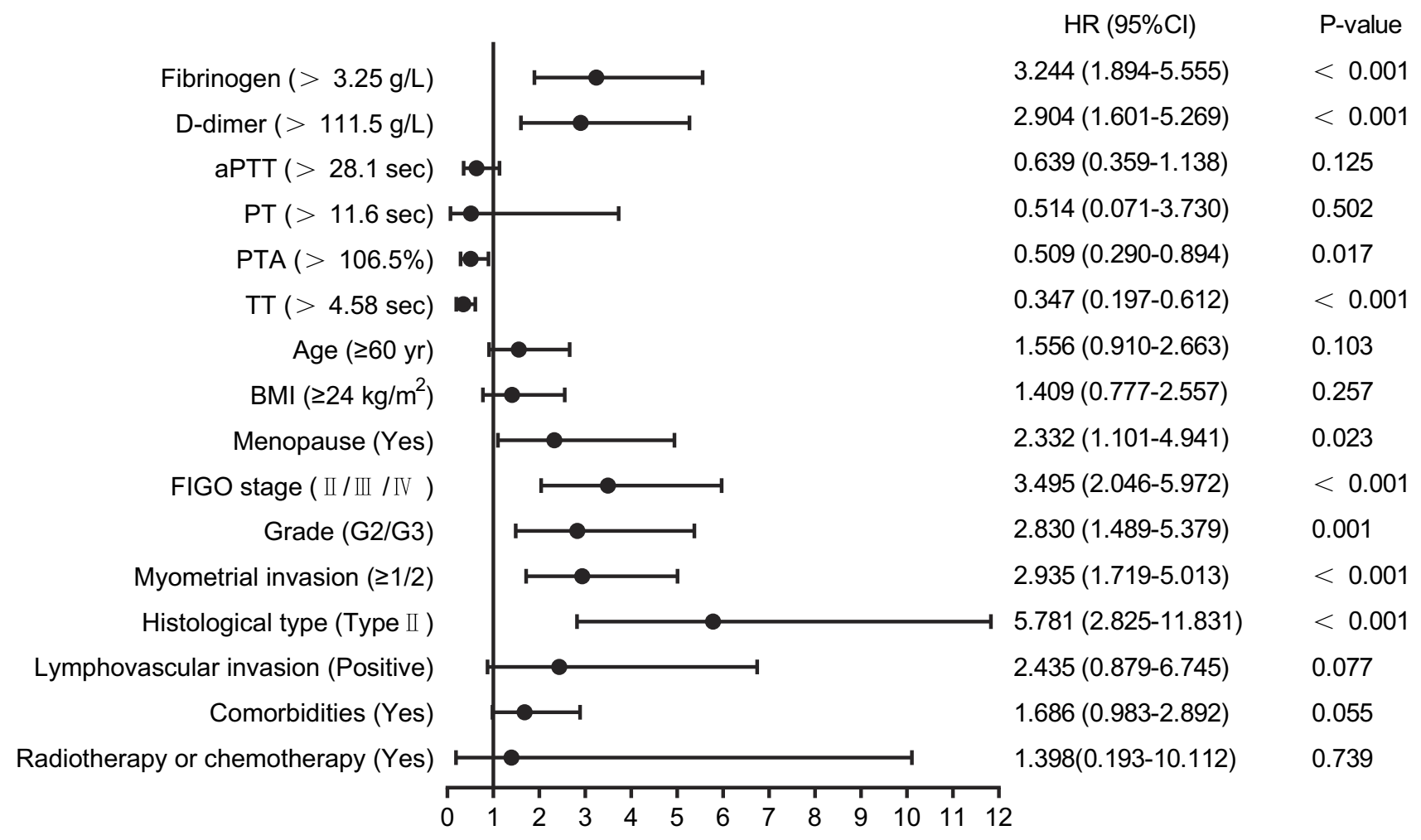

Figure 3 Univariate analysis of the OS factors.

Notes: Obtained HRs and $95 \% \mathrm{Cls}$ were compared with fibrinogen $\leq 3.25 \mathrm{~g} / \mathrm{L}$, D-dimer $\leq \mathrm{III} .5 \mathrm{~g} / \mathrm{L}$, aPTT $\leq 28.1 \mathrm{sec}, \mathrm{PT} \leq \mathrm{II} .6 \mathrm{sec}, \mathrm{PTA} \leq 106.5 \%$, TT $\leq 14.58 \mathrm{sec}$, age $<60 \mathrm{yr}$, $\mathrm{BMI}<24 \mathrm{~kg} / \mathrm{m}^{2}$, pre-menopausal status, FIGO stage I, grade GI, myometrial invasion $<\mathrm{I} / 2$, histological type I, negative lymphovascular invasion, no comorbidities and no preoperative radiotherapy and chemotherapy, respectively.

Abbreviations: HR, hazard ratio; $\mathrm{Cl}$, confidence interval; aPTT, activated partial thromboplastin time; PT, prothrombin time; PTA, prothrombin activity; TT, thrombin time; FIGO, International Federation of Gynecology and Obstetrics.

invasion and comorbidities and was confirmed to be an independent prognostic factor for EC, which is consistent with previous findings, although we found that the plasma fibrinogen level was different in grade G1 and grade G2/3 tumors, which contrasted with the conclusions of Seebacher et al and Ghezzi et al Although the relationship between fibrinogen and various clinicopathological parameters and the prognostic value of plasma fibrinogen in EC has been studied previously, only fibrinogen was reported in these studies, and other coagulation markers (D-dimer, aPTT, PTA, PT and TT) have not been discussed. The effects of BMI, menopausal status, comorbidities, and preoperative radiotherapy or chemotherapy on the prognosis of EC also should be considered. Moreover, the number of cases included in this study was 942, far exceeding the numbers in past studies. In this study, the prognostic value of six coagulation markers and more comprehensive clinical pathological parameters in EC was analyzed in more samples, and the conclusions obtained were more robust.

$\mathrm{D}$-dimer is a specific degradation product produced by the hydrolysis of fibrinolytic protein by cross-linked fibrin, and the change in plasma D-dimer levels indicates abnormal secondary fibrinolytic activity. D-dimer can be used as a biomarker to assess hyperfibrinolysis and hypercoagulability in vivo. In patients with colorectal liver metastases, a multivariate analysis showed that elevated D-dimer levels independently predicted major complications and were independently associated with reduced $\mathrm{OS}^{29}$ Cai et $\mathrm{al}^{30}$ acknowledged that the fibrinogen and D-dimer levels and the D-dimer-fibrinogen ratio (DFR) are associated with the clinical features and prognosis of gastrointestinal stromal tumors (GISTs) and that DFR may be an independent risk factor that can predict the prognosis of resectable GISTs. In a study of colorectal cancer (CRC), Guo et $\mathrm{al}^{31}$ found that the plasma D-dimer level can serve as a useful monitoring marker for distant organ metastasis in patients with CRC after therapeutic resection; Lee et $\mathrm{al}^{32}$ believed that the D-dimer level can predict the OS rate of CRC patients. Compared with patients with benign lung diseases, patients with small-cell lung cancer (SCLC) had a significantly higher plasma D-dimer level. Patients with elevated D-dimer levels before treatment had a significantly shorter progression-free survival (PFS) than those 


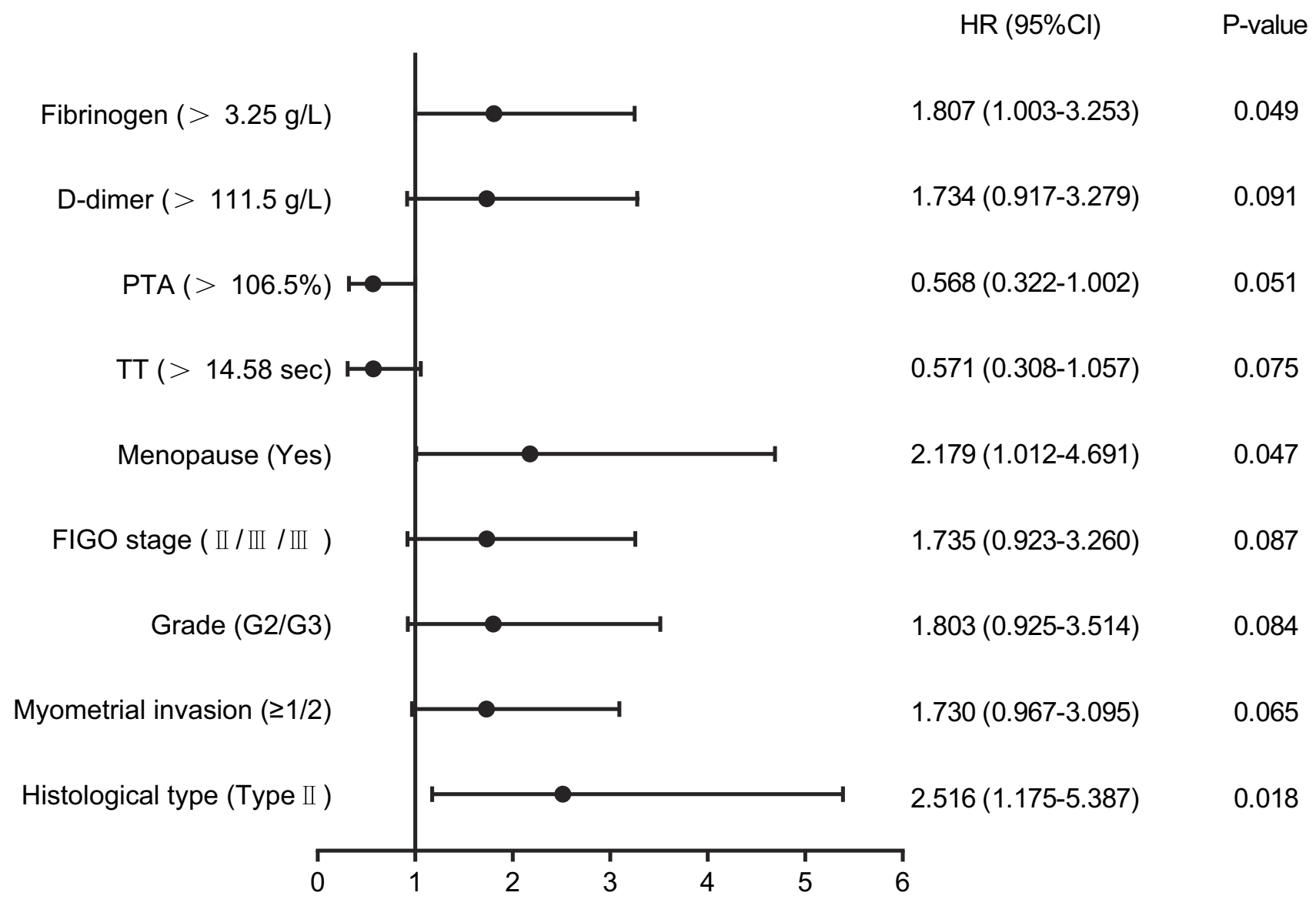

Figure 4 Multivariate analysis of the OS factors.

Notes: Obtained HRs and $95 \% \mathrm{Cls}$ were compared with fibrinogen $\leq 3.25 \mathrm{~g} / \mathrm{L}$, D-dimer $\leq \mathrm{III} .5 \mathrm{~g} / \mathrm{L}$, PTA $\leq 106.5 \%$, TT $\leq 14.58$ sec, pre-menopausal status, FIGO stage I, grade GI, myometrial invasion $<I / 2$, and histological type I, respectively.

Abbreviations: HR, hazard ratio; Cl, confidence interval; PTA, prothrombin activity; TT, thrombin time; FIGO, International Federation of Gynecology and Obstetrics.

with normal D-dimer levels; thus, preoperative plasma D-dimer levels can be used as predictors and prognostic factors for SCLC patients. ${ }^{33}$

A high D-dimer concentration has been suggested as a predictor of venous thromboembolism (VTE) in patients with $\mathrm{EC},{ }^{34}$ and the D-dimer concentration is significantly consistent with the FIGO stage and tumor grade as well as tumor recurrence and metastasis. ${ }^{35}$ Many studies have come to a conclusion that the D-dimer concentration is an independent prognostic factor for EC and is independent of VTE in the survival of patients with EC. ${ }^{36,37}$

The preoperative plasma D-dimer concentration was significantly different among patients with different ages, menopausal status, FIGO stage, tumor grade, pathologic type, depth of myometrial invasion, lymphovascular invasion and preoperative radiotherapy or chemotherapy in our study. This result is similar to those reported by other studies with other tumors and EC. The univariate analysis showed that patients with high D-dimer levels had poor survival, but the multivariate analysis showed that preoperative plasma D-dimer concentration was not an independent prognostic factor for EC. However, in the previous study, fibrinogen and D-dimer levels were not used as variables in the multivariate analysis at the same time. When the fibrinogen level was removed as a variable in this study, the results of the multivariate analysis also suggested that the $\mathrm{D}$-dimer level was an independent prognostic factor for $\mathrm{EC}(\mathrm{HR}=1.929 ; 95 \%$ $\mathrm{CI}=1.035-3.595 ; \mathrm{p}=0.039$ ). This finding indicates that whether D-dimer can independently predict the prognosis of EC remains controversial.

In our study, PT had no association with the clinicopathological characteristics and prognosis of EC. However, PTA is associated with BMI and lymphatic vascular invasion in EC. The univariate analysis found that patients with low PTA have poor survival, but the multivariate analysis showed that PTA was not an independent prognostic risk factor for EC. PTA is calculated using PT, and the different conclusions obtained in this study may be related to the 
calculation methods. PTA has the same clinical significance as PT, but PTA can reflect the activity of coagulation factors more accurately than PT. There are few studies on the relationship between PTA and cancer outcomes; although PTA cannot be used as a predictor of prognosis for EC, this factor is still a promising indicator for assessing the progression of EC.

Ge et $\mathrm{al}^{38}$ combined TT with several other preoperative indicators to predict whether patients with gastric cancer were stage IV or non-IV and correctly predicted the stage in $90.6 \%$ of patients. According to multivariate analysis in the present study, TT was significantly associated with age, menopause and FIGO stage, but not with prognosis, even though a low TT level was a negative prognostic factor for EC by univariate analysis. Thus, the link between TT and EC progression needs to be further explored.

In some studies focused on malignant tumors, patients with a reduced aPTT were found to have a poor prognosis. $^{4,39}$ This study considered that aPTT was different in patients with different menopause and comorbidities, but did not find a correlation between aPTT and the prognosis of EC.

There are still some limitations in this study, such as a portion of the patients being lost to follow-up and the inability to provide accurate information. However, we collected a large sample of patients with EC for this study, and the data from the electronic medical record system of Shengjing Hospital Affiliated to China Medical University are authentic and reliable, which guarantees the scientific nature of the research.

\section{Conclusion}

In conclusion, the fibrinogen, D-dimer, aPTT, PTA, and TT levels are associated with clinicopathological characteristics in patients with EC undergoing surgery. The preoperative fibrinogen is a valuable independent prognostic factor and is a promising biomarker for predicting the survival of patients with EC.

\section{Acknowledgments}

The study was supported by the National Natural Science Foundation of China (No. 81872123 and 81472438), Distinguished Professor of Liaoning Province, the Outstanding Scientific Fund of Shengjing Hospital (No. 201601), Liaoning Innovation Team of Colleges and Universities Support Plan, "Major Special Construction Plan" for Discipline Construction of China Medical University in 2018.

\section{Disclosure}

The authors report no conflicts of interest in this work.

\section{References}

1. Siegel RL, Miller KD, Jemal A. Cancer statistics, 2018. CA Cancer J Clin. 2018;68(1):7-30. doi:10.3322/caac.21442

2. Chen W, Zheng R, Baade PD, et al. Cancer statistics in China, 2015. CA Cancer J Clin. 2016;66(2):115-132. doi:10.3322/ caac. 21338

3. Burke William M, James O, et al; SGO Clinical Practice Endometrial Cancer Working Group. Endometrial cancer: a review and current management strategies: part I. Gynecol Oncol. 134;2014:385-392. doi:10.1016/j.ygyno.2014.05.018

4. Elena NS, Laura G, Marco L, et al. Significance and prognostic value of the coagulation profile in patients with glioblastoma: implications for personalized therapy. World Neurosurg. 2019;121:e621-e629. doi:10.1016/j.wneu.2018.09.177

5. Ke Z, Hui-Feng G, Miao M, et al. A novel scoring system based on hemostatic parameters predicts the prognosis of patients with advanced pancreatic cancer. Pancreatology. 2019;19:346-351. doi:10.1016/j. pan.2018.12.010

6. Dvorak HF. How tumors make bad blood vessels and stroma. Am J Pathol. 2003;162(6):1747-1757. doi:10.1016/S0002-9440(10)64309-X

7. Senger DR, Van deWater L, Brown LF, et al. Vascular permeability factor (VPF, VEGF) in tumor biology. Cancer Metastasis Rev. 1993;12(3-4):303-324. doi:10.1007/BF00665960

8. Dvorak HF. Vascular permeability to plasma, plasma proteins,and cells: an update. Curr Opin Hematol. 2010;17(03):225-229. doi:10.1097/MOH.0b013e3283386638

9. Verheul HMW, Hoekman K, Lupu F, et al. Platelet and coagulation activation with vascular endothelial growth factor generation in soft tissue sarcomas. Clin Cancer Res. 2000;6(1):166-171.

10. Falanga A, Marchetti M, Vignoli A. Coagulation and cancer: biological and clinical aspects. J Thromb Haemost. 2013;11:223-233. doi:10.1111/jth. 12075

11. Falanga A, Russo L, Milesi V. The coagulopathy of cancer. Curr Opin Hematol. 2014;21(5):423-429. doi:10.1097/MOH.0000000000000072

12. Burbury K. Hypercoagulopathy of cancer. Pathology. 2015;47:S35. doi:10.1097/01.PAT.0000461423.57465.ce

13. Lima LG, Monteiro RQ. Activation of blood coagulation in cancer: implications for tumour progression. Biosci Rep. 2013;33(5). doi:10.1042/BSR20130057

14. Falanga A, Schieppati F, Russo D. Cancer tissue procoagulant mechanisms and the hypercoagulable state of patients with cancer. Semin Thromb Hemost. 2015;41(07):756-764. doi:10.1055/s00000077

15. Coussens LM, Werb Z. Inflammation and cancer. Nature. 2002;420:860867. doi: $10.1038 /$ nature 01322

16. Zaino RJ, Kurman RJ, Diana KL, Morrow CP. Pathologic models to predict outcome for women with endometrial adenocarcinoma: the importance of the distinction between surgical stage and clinical stage -a Gynecologic Oncology Group study. Cancer. 1996;77:11151121. doi:10.1002/(ISSN)1097-0142

17. Steinbrecher KA, Horowitz NA, Blevins EA, et al. Colitis-associated cancer is dependent on the interplay between the hemostatic and inflammatory systems and supported by integrin alpha(M)beta(2) engagement of fibrinogen. Cancer Res. 2010;70:2634. doi:10.1158/ 0008-5472.CAN-09-3465

18. Kwaan HC, Lindholm PF. Fibrin and fibrinolysis in cancer. Semin Thromb Hemost. 2019;45:413-422.

19. Palumbo JS, Kombrinck KC, Drew AF, et al. Fibrinogen is an important determinant of the metastatic potential of circulating tumor cells. Blood. 2000;96:3320-3329. doi:10.1182/blood.V96.10.3302 
20. Verheul H, van Erp K, Homs M, et al. The relationship of vascular endothelial growth factor and coagulation factor (fibrin and fibrinogen) expression in clear cell renal cell carcinoma. Urology. 2010;75:608-614. doi:10.1016/j.urology.2009.05.075

21. Dejana E, Languino LR, Polentarutti N, et al. Interaction between fibrinogen and cultured endothelial cells. Induction ofmigration and specific binding. J Clin Invest. 1985;75(1):11-18. doi:10.1172/JCI111661

22. Ki-Tae H, Jung Kee C, Eun Youn R, et al. Prognostic influence of preoperative fibrinogen to albumin ratio for breast cancer. $J$ Breast Cancer. 2017;20:254-263. doi:10.4048/jbc.2017.20.3.254

23. Zhaowei Z, Xiaojing W, Jiange W, et al. Preoperative predictors of early death risk in bladder cancer patients treated with robot-assisted radical cystectomy. Cancer Med. 2019;8:3447-3452.

24. Peng D, Chen Z, Guohui C, et al. Combination of preoperative plasma fibrinogen and AJCC staging improves the accuracy of survival prediction for patients with stage I-II gastric cancer after curative gastrectomy. Cancer Med. 2019;8:2919-2929.

25. Uccella S, Cromi A, Vigetti D, et al. Endometrial cancer cells can express fibrinogen: immunohistochemistry and RT-PCR analysis. J Obstet Gynaecol. 2016;36:353-358. doi:10.3109/01443615.2015.1065231

26. Xingchen Z, Husheng W, Xipeng W. Preoperative CA125 and fibrinogen in patients with endometrial cancer: a risk model for predicting lymphovascular space invasion. J Gynecol Oncol. 2017;28:e11. doi:10.3802/jgo.2017.28.e11

27. Seebacher V, Polterauer S, Grimm C, et al. The prognostic value of plasma fibrinogen levels in patients with endometrial cancer: a multicentre trial. Br J Cancer. 2010;102:952-956. doi:10.1038/sj.bjc.6605547

28. Fabio G, Antonella C, Gabriele S, et al. Prognostic significance of preoperative plasma fibrinogen in endometrial cancer. Gynecol Oncol. 2010;119:309-313. doi:10.1016/j.ygyno.2010.07.014

29. Qichen C, Hong Z, Jianxiong W, et al. Preoperative D-dimer and Gamma-glutamyltranspeptidase predict major complications and survival in colorectal liver metastases patients after resection. Transl Oncol. 2019;12:996-1004. doi:10.1016/j.tranon.2019.04.011
30. Hua-Xia C, Xu-Qi L, Shu-Feng W. Prognostic value of fibrinogen and D-dimer-fibrinogen ratio in resectable gastrointestinal stromal tumors. World J Gastroenterol. 2018;24:5046-5056. doi:10.3748/wjg.v24. i44.5046

31. Yi G, Feng C, Wei C. Usefulness of plasma D-dimer level for monitoring development of distant organ metastasis in colorectal cancer patients after curative resection. Cancer Manag Res. 2018;10:4203-4216. doi:10.2147/CMAR.S177274

32. Suee L, Jae HS, Yong OS, et al. Clinical significance of coagulation factors in operable colorectal cancer. Oncol Lett. 2017;13:4669-4674. doi: $10.3892 / \mathrm{ol} .2017 .6058$

33. Cuicui Z, Yongsheng J, Yanan J, et al. Prognostic and predictive value of plasma D-dimer levels in patients with small-cell lung cancer. Int J Clin Oncol. 2018;23:1070-1075. doi:10.1007/s10147-018-1320-5

34. Cushman M, Folsom AR, Wang L, et al. Fibrin fragment D-dimer and the risk of future venous thrombosis. Blood. 2003;101:12431248. doi:10.1182/blood-2002-05-1416

35. Satoh T, Matsumoto K, Uno K, et al. Silent venous thromboembolism before treatment in endometrial cancer and the risk factors. $\mathrm{Br} J$ Cancer. 2008;99:1034-1039. doi:10.1038/sj.bjc.6604658

36. Jianpei L, Jianhua L, Yaoling L, et al. Multivariate analysis of prognostic biomarkers in surgically treated endometrial cancer. PLoS One. 2015;10:e0130640. doi:10.1371/journal.pone.0130640

37. Kohei N, Kentaro N, Masako I, et al. High pretreatment plasma D-dimer levels are related to shorter overall survival in endometrial carcinoma. Eur J Obstet Gynecol Reprod Biol. 2016;201:89-93. doi:10.1016/j.ejogrb.2016.03.041

38. Wei G, Li-Ming Z, Gang C. The combination of seven preoperative markers for predicting patients with gastric cancer to be either stage IV or non-stage IV. Gastroenterol Res Pract. 2018;2018:3450981.

39. Yongjun Q, Jingwei F. Research on the coagulation function changes in non small cell lung cancer patients and analysis of their correlation with metastasis and survival. J Buon. 2017;22:462-467.
OncoTargets and Therapy

\section{Publish your work in this journal}

OncoTargets and Therapy is an international, peer-reviewed, open access journal focusing on the pathological basis of all cancers, potential targets for therapy and treatment protocols employed to improve the management of cancer patients. The journal also focuses on the impact of management programs and new therapeutic

\section{Dovepress}

agents and protocols on patient perspectives such as quality of life adherence and satisfaction. The manuscript management system is completely online and includes a very quick and fair peer-review system, which is all easy to use. Visit http://www.dovepress.com/ testimonials.php to read real quotes from published authors. 